\title{
VARIABLE STARS AND STELLAR POPULATIONS IN ANDROMEDA XXV. III. A CENTRAL CLUSTER OR THE GALAXY NUCLEUS?*
}

\author{
Felice Cusano ${ }^{1}$, Alessia Garofalo ${ }^{1,2}$, Gisella Clementini ${ }^{1}$, Michele Cignoni ${ }^{3}$, Luciana Federici ${ }^{1}$, Marcella Marconi ${ }^{4}$, \\ Vincenzo Ripepi ${ }^{4}$, Ilaria Musella ${ }^{4}$, Vincenzo Testa ${ }^{5}$, Roberta Carini ${ }^{5}$, and Marco Faccini $^{5}$ \\ ${ }^{1}$ INAF-Osservatorio Astronomico di Bologna, Via Ranzani 1, I-40127 Bologna, Italy; felice.cusano@oabo.inaf.it \\ ${ }^{2}$ Dipartimento di Fisica e Astronomia, Università di Bologna, viale Berti Pichat, 6/2, I-40127 Bologna, Italy \\ ${ }^{3}$ Dipartimento di Fisica, Università di Pisa, Largo Bruno Pontecorvo, 3, I-56127 Pisa PI, Italy \\ ${ }^{4}$ INAF-Osservatorio Astronomico di Capodimonte, Salita Moiariello 16, I-80131 Napoli, Italy \\ ${ }^{5}$ INAF-Osservatorio Astronomico di Roma, Via di Frascati 33 I-00040 Monte Porzio Catone, Italy \\ Received 2016 May 30; revised 2016 June 30; accepted 2016 July 5; published 2016 September 19
}

\begin{abstract}
We present $B$ and $V$ time series photometry of Andromeda $\mathrm{XXV}$, the third galaxy in our program on the Andromeda's satellites, which we have imaged with the Large Binocular Cameras of the Large Binocular Telescope. The field of Andromeda XXV is found to contain 62 variable stars, for which we present light curves and characteristics of the light variation (period, amplitudes, variability type, mean magnitudes, etc.). The sample includes 57 RR Lyrae variables (46 fundamental-mode-RRab, and 11 first-overtone-RRc, pulsators), 3 anomalous Cepheids, 1 eclipsing binary system, and 1 unclassified variable. The average period of the RRab stars $(\langle\mathrm{Pab}\rangle=0.60 \sigma=0.04$ days $)$ and the period-amplitude diagram place Andromeda XXV in the class of the Oosterhoff-Intermediate objects. From the average luminosity of the RR Lyrae stars we derive for the galaxy a distance modulus of $(m-M)_{0}=24.63 \pm 0.17$ mag. The color-magnitude diagram reveals the presence in Andromeda XXV of a single, metal-poor $([\mathrm{Fe} / \mathrm{H}]=-1.8 \mathrm{dex})$ stellar population as old as $\sim 10-12 \mathrm{Gyr}$, traced by a conspicuous red giant branch and the large population of RR Lyrae stars. We discovered a spherically shaped high density of stars near the galaxy center. This structure appears to be at a distance consistent with Andromeda XXV and we suggest it could either be a star cluster or the nucleus of Andromeda XXV. We provide a summary and compare the number and characteristics of the pulsating stars in the M31 satellites analyzed so far for variability.
\end{abstract}

Key words: galaxies: dwarf - galaxies: individual (Andromeda XXV) - Local Group - stars: distances - stars: variables: general - techniques: photometric

Supporting material: data behind figure, figure set, machine-readable table

\section{INTRODUCTION}

In the framework of the $\Lambda$ cold dark matter theory, large galaxies like Andromeda (M31) are formed through the accretion and merging of smaller structures (e.g., Bullock \& Johnston 2005). The satellites that we observe today around M31 could be the surviving leftovers of the galaxy hierarchical accretion process. Their stellar contents can thus provide important hints for reconstructing the star formation history and the merging episode that led to the formation of M31. An analysis of the color-magnitude diagram (CMD) is the most powerful tool for such investigations. However, given the distance of the M31 complex, ground-based observations barely reach the bottom of the red giant branch (RGB) of these satellite galaxies and can extend down to the horizontal-branch (HB) only when $8-10 \mathrm{~m}$ class telescopes are used. For this reason, pulsating variable stars such as the RR Lyrae stars, which populate the $\mathrm{HB}$ of an old $(t \gtrsim 10 \mathrm{Gyr})$ stellar population, and the brighter and younger Cepheids, are powerful alternative tools for tracing and characterizing the different stellar generations in these systems.

This is the third paper in our series on the M31 satellites, based on $B$ and $V$ time series photometry obtained with the Large Binocular Cameras (LBC) of the Large Binocular Telescope (LBT). We characterize the resolved stellar

\footnotetext{
* Based on data collected with the Large Binocular Cameras at the Large Binocular Telescope.
}

populations of the M31 companions using the CMD and the properties of variable stars and from this derive hints on the nature, origin, and fate of Andromeda's satellites in the global context of merging and accretion episodes occurring in M31. Details on the survey and results from the study of Andromeda XIX (And XIX) and Andromeda XXI (And XXI) were presented in Cusano et al. (2013, Paper I) and Cusano et al. (2015, Paper II), respectively. In this paper we report results from the study of Andromeda XXV (And XXV).

And XXV was discovered by Richardson et al. (2011) in the context of the PAndAS survey (Martin et al. 2013 and the references therein) and later found to be a member of the thin plane of satellites identified in M31 by Ibata et al. (2013). The discovery paper reports a distance modulus of $(m-M)_{0}=$ $24.55 \pm 0.12 \mathrm{mag}$ for And XXV from the luminosity of the galaxy $\mathrm{HB}$ and a half-light radius $\left(r_{h}\right)$ of 3'.0 (corresponding to $r_{h}=732 \pm 60 \mathrm{pc}$ at the distance of And XXV). Later, Conn et al. (2012), adopting a bayesian approach to locate the tip of the RGB, revised the distance modulus of And XXV to $(m-M)_{0}=24.33_{-0.21}^{+0.07} \mathrm{mag}$. This is more than $1 \sigma$ shorter than Richardson et al.'s (2011) distance based on the HB luminosity. And XXV has a metallicity of $[\mathrm{Fe} / \mathrm{H}]=-1.9 \pm 0.1 \mathrm{dex}$ (Collins et al. 2013) as estimated from the Calcium triplet (Ca II) of the galaxy red giants. The same authors measured a velocity dispersion of $\sigma=3.0_{-1.1}^{+1.2} \mathrm{~km} \mathrm{~s}^{-1}$ from 25 spectroscopically confirmed members. This value is rather low, when compared with the large extension of And XXV. Collins et al. (2013) also 
Table 1

Log of And XXV Observations

\begin{tabular}{lcrcc}
\hline \hline Dates & Filter & $N$ & $\begin{array}{c}\text { Exposure Time } \\
(\mathrm{s})\end{array}$ & $\begin{array}{c}\text { Seeing (FWHM) } \\
(\operatorname{arcsec})\end{array}$ \\
\hline 2011 Oct 18 & $B$ & 1 & 400 & 1 \\
2011 Oct 20-24 & $B$ & 84 & 400 & $0.8-1$ \\
2011 Oct 18 & $V$ & 3 & 400 & 1 \\
2011 Oct 20-24 & $V$ & 84 & 400 & $0.8-1$ \\
\hline
\end{tabular}

estimate a mass-to-light ratio inside one $r_{h}$ of $[M / L]_{r_{h}}=$ $10.3 M_{\odot} / L_{\odot}$ that, according to the authors, is consistent with a stellar population without a dark matter component. In a more recent paper Collins et al. (2014), using the circular velocity within the galaxy half-light radius as an argument, concluded that the mass of And XXV should have been much more prominent in the past, for the galaxy to be able to form stars. On the other hand, the low-velocity dispersion of And XXV's member stars would naturally arise in the context of the MOND theory (McGaugh \& Milgrom 2013).

The paper is organized as follows: observations, data reduction, and calibration of And XXV photometry are presented in Section 2. Results on the identification and characterization of the variable stars, the catalog of light curves, and the Oosterhoff classification of And XXV are discussed in Section 3. The distance to And XXV derived from the RR Lyrae stars is presented in Section 4. The galaxy CMD, along with the spatial distribution of the various stellar components, are discussed in Section 5. Section 6 examines the discovery of a spherically shaped high density of stars close to the galaxy center. The main results of the present study are summarized in Section 7.

\section{OBSERVATIONS AND DATA REDUCTION}

A total of $85 B$ and $87 \mathrm{~V}$ images, each of $400 \mathrm{~s}$ exposure, were obtained with the LBC of a region of $23^{\prime} \times 23^{\prime}$ centered on And XXV (R.A. $=00^{\mathrm{h}} 30^{\mathrm{m}} 08^{\mathrm{s}} .9$, decl. $=+46^{\circ} 51^{\prime} 07^{\prime \prime}$, J2000.0; Richardson et al. 2011) from the 18th to the 24th of 2011 October. Observations in the $B$-band were acquired with the blue camera and simultaneous $V$ imaging were acquired with the red camera of the LBC. All images were obtained under favorable conditions of seeing $\leqslant 1^{\prime \prime}$. The $\log$ of the observations of And XXV is provided in Table 1.

Photometric reduction of And XXV imaging was carried out following the same procedure as described in detail in Paper I (And XIX) and II (And XXI). The PSF photometry was performed using the DAOPHOT - ALLSTAR - ALLERAME packages (Stetson 1987, 1994). The Landolt standard fields L92 and SA113, observed during the run, were used to derive calibration equations and to tie the DAOPHOT magnitudes to the Johnson standard system. The new calibration equations 6 are totally consistent with those derived in Paper I, once differences in airmass and exposure times are accounted for.

\section{VARIABLE STARS}

The search for variable stars was carried out by starting from the variability index computed in DAOMASTER (Stetson 1994). The candidate variables were then analyzed by studying their

\footnotetext{
$6 \quad B-b=27.696-0.113 \times(b-v) \mathrm{rms}=0.03$, $V-v=27.542-0.060 \times(b-v) \mathrm{rms}=0.03$.
}

$B$-band and $V$-band light curves with the Graphical Analyzer of Time Series (GRATIS), private software developed at the Bologna Observatory by P. Montegriffo (see, e.g., Clementini et al. 2000). More details on this procedure can be found in Paper I. In the $B, V$ data sets of And XXV we identified a total of 62 variable stars: 57 RR Lyrae stars (see Section 3.1), 3 anomalous Cepheids (AC; see Section 3.2), 1 eclipsing binary (ECL), and 1 unclassified variable. The properties of the confirmed variable stars in And XXV are summarized in Table 2 . We ordered the variables by increasing number based on the proximity to the galaxy center, adopting the coordinates by Richardson et al. (2011). Column 1 lists the star identifier, and Columns 2 and 3 give the right ascension and declination (J2000 epoch), respectively, obtained from our astrometrized catalogs. Column 4 provides the type of variability. A question mark identifies stars whose classifications are uncertain. Columns 5 and 6 list the period and the Heliocentric Julian Day of maximum light, respectively. Columns 7 and 8 give the intensity-weighted mean $B$ and $V$ magnitudes, while Columns 9 and 10 list the corresponding amplitudes of the light variation. The two solid lines in the table separate the variable stars within the area enclosed once and twice the galaxy $r_{h}$. Example light curves for RR Lyrae and other types of variables in And XXV are shown in Figure 1. The full catalog of light curves is available only in the electronic version.

\subsection{RR Lyrae Stars}

In the field of And XXV we have identified a total of $57 \mathrm{RR}$ Lyrae stars: 46 fundamental-mode (RRab) and 11 first-overtone (RRc) stars. Of them, 56 likely belong to And XXV, whereas V63 is $\sim 12$ arcmin away from the center of the galaxy and is most likely an RR Lyrae of the M31 halo. We do not include this star when deriving properties of And XXV such as the distance, the Oosterhoff type, etc. Figure 2 shows the period distribution of the RR Lyrae stars. The average period of the whole sample of 45 RRab stars in And XXV is $\left\langle P_{\mathrm{ab}}\right\rangle=0.61$ day ( $\sigma=0.05$ day) and becomes $\left\langle P_{\mathrm{ab}}\right\rangle=0.60$ day ( $\sigma=0.05$ day) if we only consider 15 RRab'(s) within the $r_{h}$ convolved with the galaxy ellipticity, while it is $\left\langle P_{\mathrm{ab}}\right\rangle=0.60$ day ( $\sigma=0.05$ day) for the 32 RRabs within twice the $r_{h}$. In any case, And XXV is classified as an Oosterhoff-Intermediate (Oo Int) system (Oosterhoff 1939; Catelan 2009).

The fraction of RRc stars in And XXV over the whole number of RR Lyrae stars is $f_{c}=N_{c} / N_{\mathrm{ab}+\mathrm{c}}=0.19 \pm 0.07$, which is intermediate between what is expected for Oosterhoff II (Oo II; $f_{c} \sim 0.44$ ) and Oosterhoff I (Oo I; $f_{c} \sim 0.17$ ) systems (Catelan 2009), again confirming the classification of And XXV as Oo Int.

The period-amplitude diagram (also known as a Bailey diagram, Bailey 1902) of the RR Lyrae stars in And XXV is shown in the left panel of Figure 3, together with the loci defined by RR Lyrae stars in the Oo I Galactic globular cluster M3 (lower line) and the Oo II globular cluster $\omega$ Cen (upper line), according to Clement \& Rowe (2000). We separated the RR Lyrae stars in three groups depending on the position inside once and twice the area delimited by the $r_{h}$ convolved with the galaxy ellipticity, and in the whole LBC field of view (FOV). Almost all RR Lyrae stars fall on the Oo I locus or between the two lines, but closer to the Oo I position. Only three RR Lyrae stars are near the Oo II locus. In the right panel of Figure 3 the period-amplitude diagram of And XXV's RR Lyrae stars is compared to the distribution of RR Lyrae in And XIX, 
Table 2

Identification and Properties of the Variable Stars Detected in And XXV

\begin{tabular}{|c|c|c|c|c|c|c|c|c|c|}
\hline Name & $\begin{array}{c}\alpha \\
(2000)\end{array}$ & $\begin{array}{c}\delta \\
(2000)\end{array}$ & Type & $\begin{array}{c}\mathrm{P} \\
\text { (days) }\end{array}$ & $\begin{array}{c}\text { Epoch }(\max ) \\
\text { HJD }(-2455000)\end{array}$ & $\begin{array}{c}\langle B\rangle \\
(\mathrm{mag})\end{array}$ & $\begin{array}{c}\langle V\rangle \\
(\mathrm{mag})\end{array}$ & $\begin{array}{c}A_{B} \\
(\mathrm{mag})\end{array}$ & $\begin{array}{c}A_{V} \\
(\mathrm{mag})\end{array}$ \\
\hline V1 & $00: 30: 10.688$ & $+46: 51: 21.71$ & RRab & 0.6078 & 852.561 & 25.90 & 25.42 & 1.10 & 0.86 \\
\hline $\mathrm{V} 2$ & 00:30:09.937 & $+46: 51: 34.93$ & RRab & 0.5459 & 851.356 & 25.77 & 25.38 & 1.42 & 0.87 \\
\hline V3 & 00:30:09.306 & $+46: 51: 43.73$ & RRab & 0.6147 & 852.048 & 25.80 & 25.43 & 1.42 & 0.86 \\
\hline V4 & $00: 30: 12.324$ & $+46: 50: 58.88$ & $\mathrm{AC}$ & 1.355 & 854.800 & 24.23 & 23.79 & 0.68 & 0.56 \\
\hline V5 & 00:30:05.194 & $+46: 50: 48.71$ & $\mathrm{RRc}$ & 0.3858 & 851.920 & 25.82 & 25.41 & 0.72 & 0.36 \\
\hline V6 & $00: 30: 11.050$ & $+46: 51: 58.73$ & RRab & 0.609 & 854.732 & 25.58 & 25.23 & 0.98 & 0.76 \\
\hline V7 & 00:30:05.122 & $+46: 50: 27.28$ & RRab & 0.6116 & 857.708 & 25.80 & 25.39 & 1.00 & 0.72 \\
\hline V8 & 00:30:05.122 & $+46: 50: 27.28$ & $\mathrm{RRc}$ & 0.3457 & 851.164 & 25.53 & 25.17 & 0.73 & 0.70 \\
\hline V9 & $00: 30: 14.345$ & $+46: 50: 47.45$ & RRab & 0.5742 & 856.642 & 25.57 & 25.15 & 1.41 & 1.10 \\
\hline V10 & 00:30:04.745 & $+46: 50: 06.57$ & RRab & 0.72 & 854.705 & 25.71 & 25.32 & 0.76 & 0.49 \\
\hline V11 & $00: 30: 14.186$ & $+46: 50: 17.44$ & RRab & 0.5963 & 856.705 & 25.68 & 25.33 & 1.06 & 0.98 \\
\hline V12 & $00: 30: 12.939$ & $+46: 52: 22.79$ & RRab & 0.5466 & 854.795 & 25.73 & 25.39 & 1.44 & 1.10 \\
\hline V13 & $00: 30: 02.642$ & $+46: 50: 12.32$ & RRab & 0.7254 & 852.133 & 25.80 & 25.38 & 1.00 & 0.78 \\
\hline V14 & 00:30:02.612 & $+46: 52: 23.61$ & RRab & 0.576 & 854.804 & 25.72 & 25.33 & 1.36 & 1.06 \\
\hline V15 & $00: 30: 14.464$ & $+46: 49: 28.74$ & RRab & 0.591 & 856.663 & 25.63 & 25.20 & 1.01 & 0.79 \\
\hline V16 & $00: 30: 17.886$ & $+46: 51: 58.24$ & RRab & 0.610 & 851.573 & 25.95 & 25.53 & 0.90 & 0.56 \\
\hline V17 & $00: 30: 18.136$ & $+46: 50: 05.58$ & RRab & 0.5675 & 855.784 & 25.68 & 25.31 & 1.36 & 1.18 \\
\hline V18 & $00: 30: 02.457$ & $+46: 48: 57.10$ & $\mathrm{RRc}$ & 0.375 & 854.810 & 25.64 & 25.31 & 0.74 & 0.33 \\
\hline V19 & $00: 30: 13.024$ & $+46: 48: 31.33$ & RRab & 0.577 & 855.587 & 25.66 & 25.28 & 1.22 & 0.95 \\
\hline V20 & $00: 29: 59.216$ & $+46: 52: 40.02$ & $\mathrm{RRc}$ & 0.3085 & 852.695 & 25.43 & 25.16 & 0.53 & 0.36 \\
\hline V21 & $00: 30: 21.242$ & $+46: 51: 02.77$ & RRab & 0.562 & 854.804 & 25.41 & 25.07 & 1.60 & 1.25 \\
\hline $\mathrm{V} 22$ & $00: 29: 58.666$ & $+46: 53: 04.62$ & RRab & 0.655 & 857.894 & 25.69 & 25.31 & 0.71 & 0.65 \\
\hline V23 & $00: 30: 21.905$ & $+46: 51: 36.72$ & $\mathrm{RRc}$ & 0.3668 & 858.900 & 25.49 & 25.15 & 0.81 & 0.61 \\
\hline V24 & $00: 30: 18.610$ & $+46: 53: 37.02$ & RRab & 0.537 & 854.816 & 25.66 & 25.29 & 1.89 & 1.28 \\
\hline V25 & $00: 30: 15.060$ & $+46: 47: 56.28$ & RRab & 0.637 & 856.670 & 25.73 & 25.27 & 0.80 & 0.51 \\
\hline V26 & $00: 30: 23.121$ & $+46: 51: 10.67$ & $\mathrm{RRc}$ & 0.405 & 856.782 & 25.61 & 25.26 & 0.70 & 0.47 \\
\hline V27 & $00: 30: 21.141$ & $+46: 49: 12.98$ & RRab & 0.547 & 857.652 & 25.60 & 25.22 & 1.25 & 0.97 \\
\hline V28 & $00: 30: 17.693$ & $+46: 48: 10.12$ & RRab & 0.565 & 856.747 & 25.62 & 25.25 & 1.42 & 1.12 \\
\hline V29 & $00: 29: 55.136$ & $+46: 49: 28.51$ & RRab & 0.632 & 856.860 & 25.73 & 25.26 & 0.781 & 0.56 \\
\hline V30 & 00:30:03.691 & $+46: 47: 25.31$ & $\mathrm{RRc}$ & 0.368 & 856.767 & 25.55 & 25.20 & 0.452 & 0.41 \\
\hline V31 & $00: 30: 13.107$ & $+46: 47: 15.13$ & RRab & 0.669 & 856.780 & 25.50 & 24.88 & 0.845 & 0.51 \\
\hline V32 & $00: 30: 11.748$ & $+46: 55: 04.50$ & $\mathrm{RRc}$ & 0.378 & 856.677 & 25.60 & 25.34 & 0.66 & 0.59 \\
\hline V33 & $00: 29: 58.771$ & $+46: 47: 48.94$ & RRab & 0.577 & 856.850 & 25.61 & 25.24 & 0.87 & 0.72 \\
\hline V34 & $00: 30: 26.025$ & $+46: 51: 13.58$ & RRab & 0.5575 & 858.894 & 25.65 & 25.30 & 1.16 & 0.81 \\
\hline V35 & $00: 30: 19.772$ & $+46: 47: 45.21$ & RRab & 0.579 & 854.780 & 25.64 & 25.17 & 1.50 & 1.17 \\
\hline V36 & $00: 30: 26.764$ & $+46: 50: 36.83$ & RRab & 0.5678 & 858.615 & 25.47 & 25.14 & 1.71 & 1.32 \\
\hline V37 & 00:30:02.239 & $+46: 46: 45.76$ & RRab & 0.6992 & 857.747 & 25.64 & 25.23 & 0.80 & 0.62 \\
\hline V38 & $00: 30: 18.484$ & $+46: 47: 02.00$ & RRab & 0.67 & 856.802 & 25.63 & 25.30 & 0.72 & 0.47 \\
\hline V39 & $00: 30: 22.544$ & $+46: 54: 27.27$ & RRab & 0.5766 & 858.601 & 25.54 & 25.22 & 1.21 & 1.00 \\
\hline V40 & $00: 30: 18.502$ & $+46: 46: 50.06$ & RRab & 0.621 & 858.638 & 25.66 & 25.27 & 1.37 & 0.82 \\
\hline V41 & $00: 29: 55.859$ & $+46: 47: 19.20$ & RRab & 0.618 & 856.664 & 25.66 & 25.29 & 0.82 & 0.67 \\
\hline V43 & $00: 29: 48.512$ & $+46: 51: 50.74$ & $\mathrm{RRc}$ & 0.37 & 855.660 & 25.59 & 25.17 & 0.64 & 0.34 \\
\hline V44 & $00: 29: 48.589$ & $+46: 49: 46.67$ & RRab & 0.615 & 858.598 & 25.59 & 25.25 & 0.92 & 0.69 \\
\hline V45 & $00: 30: 04.316$ & $+46: 44: 45.19$ & RRab & 0.5867 & 855.842 & 25.59 & 25.33 & 1.13 & 0.93 \\
\hline V46 & $00: 29: 42.803$ & $+46: 51: 51.83$ & $\mathrm{AC} / \mathrm{CC}$ ? & 1.213 & 852.697 & 23.82 & 23.38 & 0.62 & 0.40 \\
\hline V47 & 00:30:08.635 & $+46: 44: 29.81$ & $\mathrm{RRab}$ & 0.6322 & 854.715 & 25.65 & 25.36 & 1.02 & 0.81 \\
\hline V48 & $00: 29: 57.962$ & $+46: 44: 56.31$ & RRab & 0.6325 & 857.792 & 25.74 & 25.36 & 0.81 & 0.70 \\
\hline V49 & $00: 30: 25.549$ & $+46: 56: 26.38$ & RRab & 0.592 & 855.790 & 25.65 & 25.33 & 1.05 & 0.92 \\
\hline V50 & $00: 29: 41.661$ & $+46: 50: 24.07$ & RRab & 0.579 & 856.863 & 25.65 & 25.45 & 0.81 & 0.67 \\
\hline V51 & $00: 30: 17.641$ & $+46: 57: 49.18$ & RRab & 0.6371 & 855.593 & 25.43 & 25.08 & 1.13 & 0.93 \\
\hline V52 & 00:30:09.862 & $+46: 43: 59.74$ & RRab & 0.6243 & 856.652 & 25.58 & 25.20 & 0.97 & 0.71 \\
\hline V53 & 00:30:20.789 & $+46: 44: 32.70$ & $\mathrm{RRc}$ ? & 0.3934 & 854.826 & 25.54 & 25.26 & 0.56 & 0.42 \\
\hline V54 & $00: 30: 24.470$ & $+46: 57: 13.95$ & RRab & 0.635 & 855.580 & 25.66 & 25.20 & 0.80 & 0.65 \\
\hline V55 & $00: 30: 37.347$ & $+46: 52: 57.46$ & RRab & 0.681 & 851.572 & 25.61 & 25.231 & 0.80 & 0.66 \\
\hline V56 & $00: 29: 50.346$ & $+46: 44: 58.80$ & $\mathrm{RRc}$ & 0.299 & 858.907 & 25.66 & 25.39 & 0.67 & 0.53 \\
\hline V57 & $00: 30: 16.060$ & $+46: 42: 25.70$ & RRab & 0.57 & 856.764 & 25.77 & 25.38 & 0.97 & 0.72 \\
\hline V58 & $00: 29: 32.588$ & $+46: 49: 37.73$ & $\mathrm{AC}$ & 0.531 & 855.810 & 24.53 & 24.24 & 0.90 & 0.76 \\
\hline V59 & $00: 29: 56.648$ & $+46: 42: 10.13$ & RRab & 0.642 & 856.847 & 25.45 & 25.22 & 1.201 & 1.087 \\
\hline $\mathrm{V} 60^{\mathrm{a}}$ & $00: 30: 13.652$ & $+46: 41: 30.72$ & uncl & 0.848 & 854.595 & 21.48 & 19.96 & 0.239 & 0.197 \\
\hline $\mathrm{V} 61^{\mathrm{a}}$ & $00: 30: 06.316$ & $+46: 41: 26.44$ & ECL & 0.25677 & 856.760 & 24.24 & 23.22 & 1.39 & 0.886 \\
\hline V62 & $00: 30: 14.030$ & $+46: 41: 22.26$ & RRab & 0.5337 & 856.732 & 25.67 & 25.22 & 1.58 & 1.233 \\
\hline $\mathrm{V} 63^{\mathrm{a}}$ & $00: 29: 49.898$ & $+46: 49: 07.68$ & RRab & 0.5486 & 855.629 & 25.55 & 25.16 & 1.483 & 1.157 \\
\hline
\end{tabular}

Note. Solid lines separate variable stars located within once and twice the galaxy half-light radius.

${ }^{\text {a }}$ Field variable stars.

(This table is available in machine-readable form.) 
B
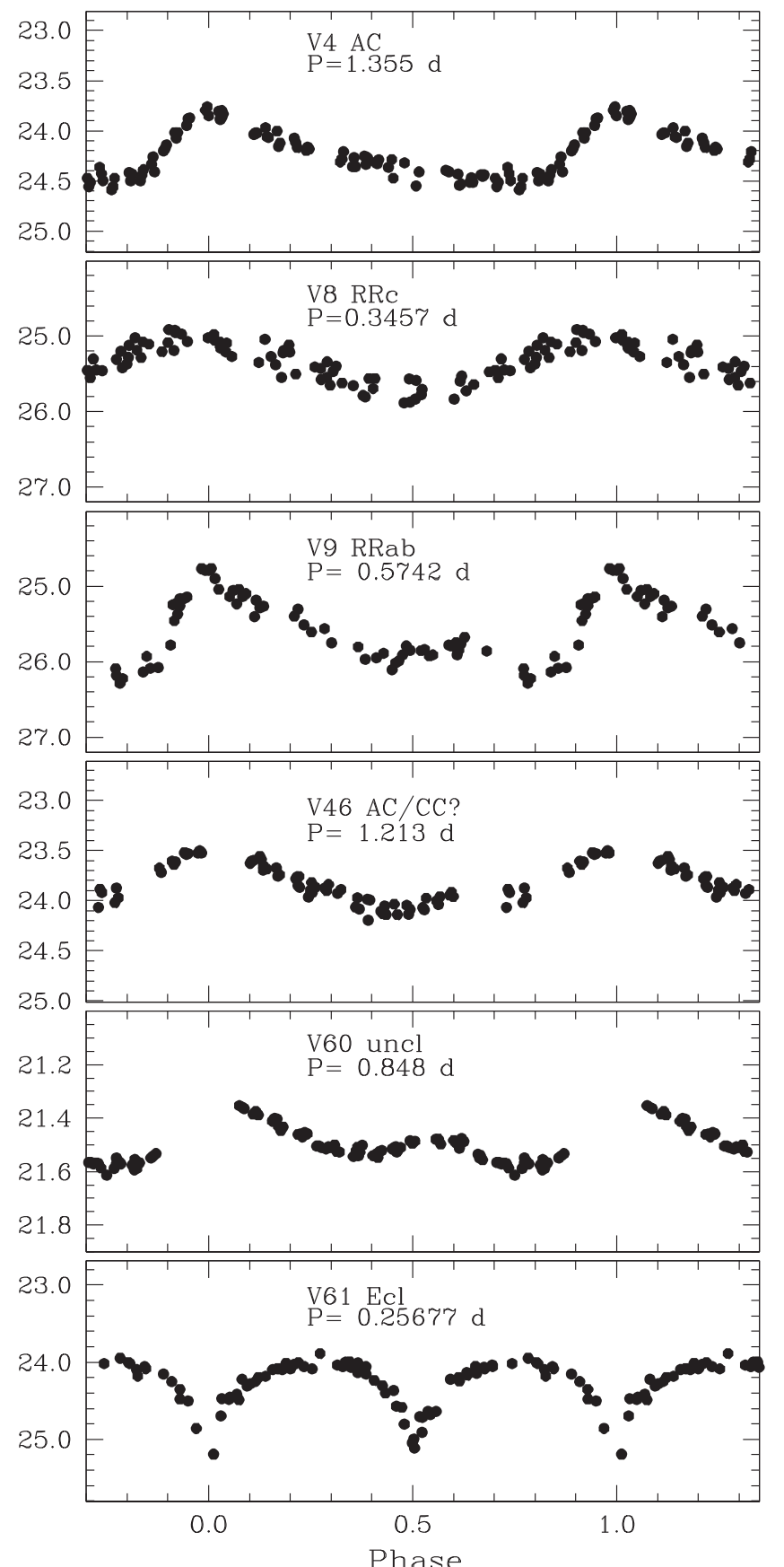

$\mathrm{V}$

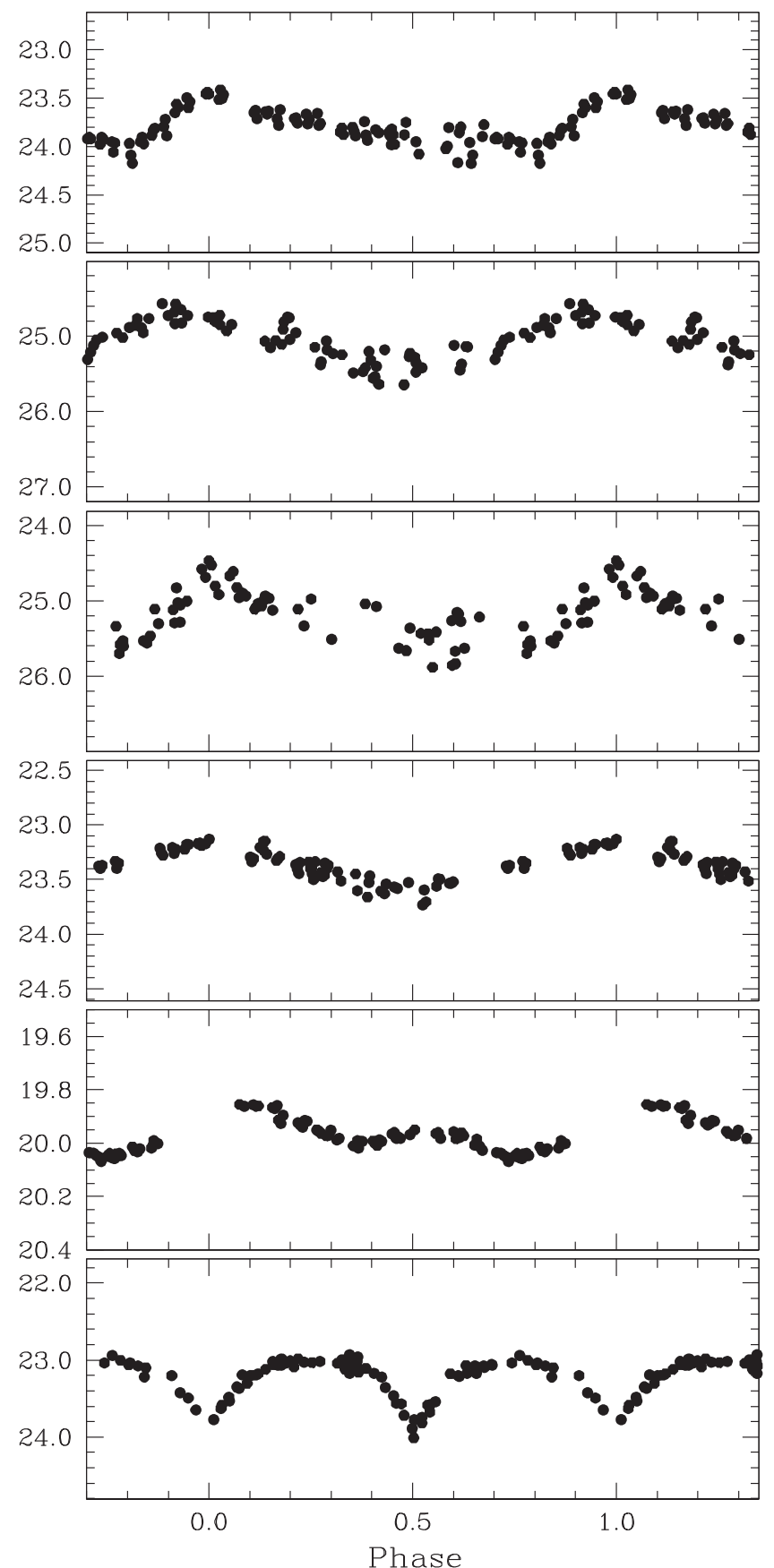

Figure 1. Examples of $B$-band (left panels) and $V$-band (right panels) light curves for different types of variable stars identified in And XXV. Typical internal errors of the single-epoch data range from 0.01 at $B \sim 21.30 \mathrm{mag}$ (corresponding to the maximum light of the unclassified variable V60) to $0.30 \mathrm{mag}$ at $B \sim 26.20 \mathrm{mag}$ (corresponding to the minimum light of the fundamental-mode RR Lyrae V9), and similarly, from $0.01 \mathrm{mag}$ at $V \sim 19.90 \mathrm{mag}$ to $0.30 \mathrm{mag}$ at $V \sim 26.00 \mathrm{mag}$. The BV photometry shown for each light curve is available as Data behind the Figure. The data used to create this figure are available.

(The complete figure set (9 images) is available.)

And XXI, and in three halo fields at 4, 6, (Sarajedini et al. 2009) and $35 \mathrm{kpc}$ (Brown et al. 2004) from the center of M31, respectively. The RR Lyrae stars in the M31 halo conform more to the Oo I type, while the dwarf Spheroidal galaxy (dSph) satellites are more Oo Int, with a slight trend toward Oo I type, which decreases with increasing distance from the M31 center.

The RR Lyrae stars trace the old populations of a galaxy and from their positions it is thus possible to draw a first picture of the geometry of And XXV. The spatial distribution of the RR Lyrae stars in And XXV is shown in Figure 4 (filled red circles), and reveals the elongated structure of this galaxy. Adopting the structural parameters derived by Richardson et al. (2011), many RR Lyrae stars appear to be placed beyond the galaxy half-light radius, traced by the inner black ellipse, and create a protrusion of stars that extends beyond twice the $r_{h}$. In particular, more than half of the RR Lyrae stars (37 out of 56) are located southward with respect to the galaxy center and 


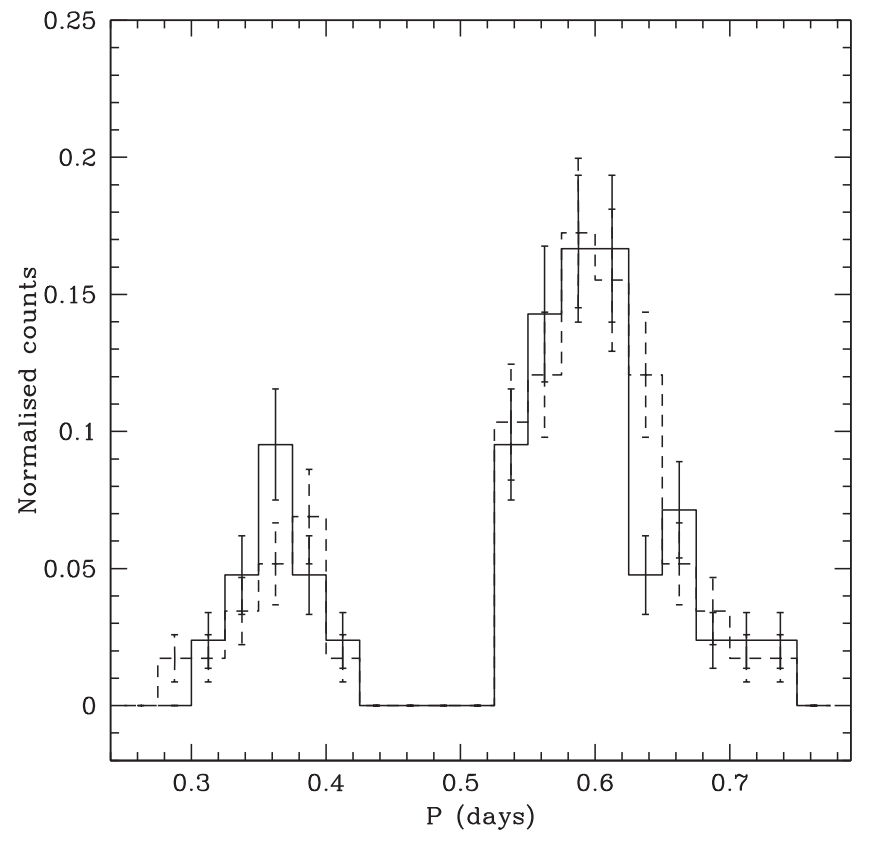

Figure 2. The dashed histogram represents the distribution of the periods of all the RR Lyrae stars identified in And XXV. The continuous line histogram is for RR Lyrae enclosed in twice the galaxy $r_{h}$. The bin size is 0.025 days.

suggest a stretched distribution of And XXV'(s) stars. Most of them are aligned along the direction toward M31.

\subsection{Anomalous Cepheids}

In And XXV we identified three variable stars, namely, V4, V46, and V58, which fall inside the instability strip (see Marconi et al. 2004), but have luminosities exceeding the average magnitude of the RR Lyrae stars by about 1 mag. Following the procedure discussed in Papers I and II, by the cross study with isochrones in the CMD, the period-Wesenheit (PW) relations for ACs and classical Cepheids (CCs), and the analysis of the light curves, we classify these three stars as ACs. However the classification for the star V46 is more uncertain and this star can be also seen as a short period CC.

To obtain the Wesenheit index of these three variables we subtracted the color term multiplied by 3.1 (adopting the Cardelli et al. 1989, law for extinction) to the absolute magnitudes derived by their intensity-averaged apparent visual magnitudes, $\langle V\rangle$, adopting the distance modulus of $(m-$ $M)_{0}=24.63 \pm 0.17 \mathrm{mag}$ as derived from the RR Lyrae stars (see Section 4). In the left panel of Figure 5 are displayed the PW relations for ACs in the Large Magellanic Cloud (LMC, solid lines) derived by Ripepi et al. (2014). ${ }^{7}$ V4, V46, and V58 fall within $1 \sigma$ of the Ripepi et al. (2014) relations for fundamental-mode and first-overtone pulsators. In the right panel of Figure 5 we compare the three variables with the PW relations of the LMC CCs by Jacyszyn-Dobrzeniecka et al. (2016). Clearly, V4 does not fit the CC PW relations, while both V46 and V58 are within $1 \sigma$ of the fundamental-mode CC PW. However, looking at the shape of the light curve V58 shows the typical trend variation of an AC (see Papers I and II for examples). Therefore, we finally classify V58 as AC, while for V46 we give an intermediate classification between $\mathrm{AC}$ and

\footnotetext{
7 Ripepi et al.'s (2014) relations were derived for the $V$-bands and $I$-bands and we have converted them to $B$ and $V$ using Equation (12) of Marconi et al. (2004).
}

CC. Since both V46 and V58 are outside twice the area defined by the galaxy $r_{h}$, they could either belong to a background/ foreground feature of the M31 field, or could be recently formed stars due to a peripheral episode of star formation in And XXV triggered by tidal interaction. In any case, given the uncertain membership of V46 and V58, we have computed the specific frequency of ACs in And XXV under the assumption that V4 is the only AC belonging to the galaxy. This is shown in Figure 6 together with the AC-specific frequency in a number of Milky Way (MW) and M31 dwarf satellites. And XXV does not follow the relation traced by the other galaxies. Moreover, to make a more consistent comparison between the ACs discovered in this paper, in Papers I and II, and those presented in other studies (see Pritzl et al. 2005 and the references therein), we have revised the AC-specific frequency in dwarf galaxies analyzed in those previous studies, considering only those ACs falling within $1 \sigma$ of the PW relations for as bona fide ACs. This tool has confirmed the AC number in the following satellites: Leo I, Leo II, Draco, Ursa Minor, Carina, Sculptor, and Fornax but has reduced it in And I and And II (from 1 to 0), And III (from 5 to 2), And VI (from 6 to 4), and Sextans (from 4 to 2). The red solid lines plotted in both panels of Figure 6 are the least-square fits obtained by adopting the revised specific frequencies computed as discussed above. They are described by the following equations:

$$
\log S=0.23( \pm 0.57) \times M v+2.22( \pm 0.57)
$$

$\log S=-1.39( \pm 0.44) \times[\mathrm{Fe} / \mathrm{H}]-2.62( \pm 0.83)$.

Black lines mark instead the least-square fits originally obtained in Pritzl et al. (2005).

Gray dashed arrows indicate the position that the revised galaxies would occupy in these plots if we used their ACspecific frequencies from the literature. In the right panel, new and previous fits are in perfect agreement, while in the left panel the slope of the new fit is less steep, indicating that for fainter galaxies a smaller number of ACs is expected.

\subsection{Other Variables}

In the field of And XXV we discovered two variable stars, namely, V60 and V61, which in the CMD (Figure 7) are outside the instability strip for Cepheids and RR Lyrae stars (Marconi et al. 2004). V61 was classified by the light curve analysis as an ECL, while we were unable to give a clear classification for V60. The position in the CMD (see Figure 7) and the projected spatial distribution (Figure 4) suggest them as more likely belonging to the M31 field or to MW. V60 in particular, with a $B-V \sim 1.5 \mathrm{mag}$ and a $V$ of $20 \mathrm{mag}$, is a field star, beyond any doubt.

\section{DISTANCE}

The $V$ average magnitude of the whole sample of RR Lyrae stars in And XXV is $\langle V(\mathrm{RR})\rangle=25.26 \pm 0.10 \mathrm{mag}$ (on average 56 stars). If we exclude V31, which is 0.39 mag brighter than the RR Lyrae average magnitude and can be either a blended star or an evolved RR Lyrae, we obtain $\langle V(\mathrm{RR})\rangle=25.27 \pm 0.09$ mag. Considering instead the subsample of RR Lyrae inside an area delimited by And XXV $r_{h}$ the average $V$ magnitude is $\langle V(\mathrm{RR})\rangle=25.33 \pm 0.09 \mathrm{mag}$ (average on 18 stars). As for Papers I and II, to correct for interstellar extinction we derived the reddening from the galaxy RR Lyrae stars, adopting the method 

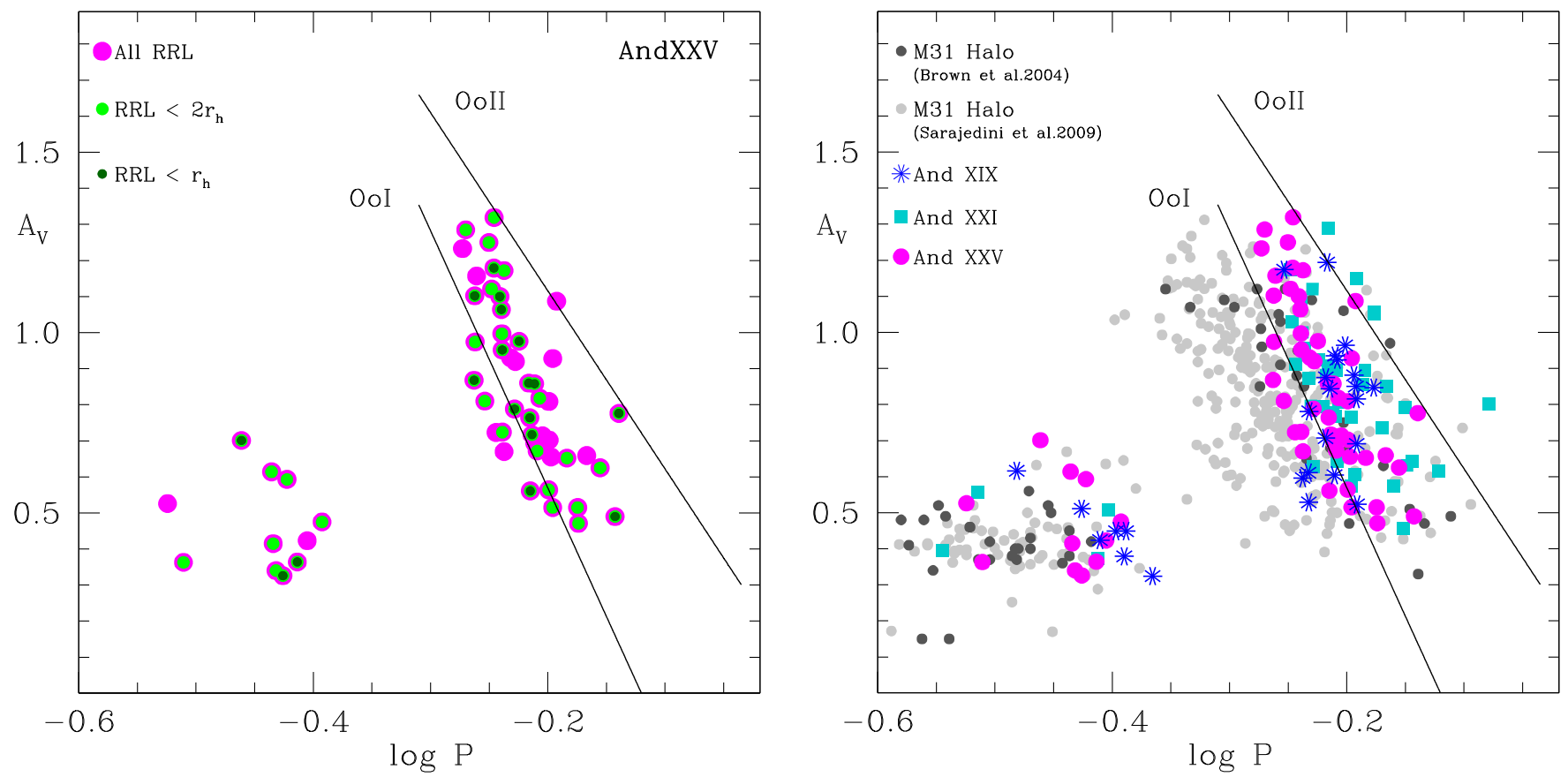

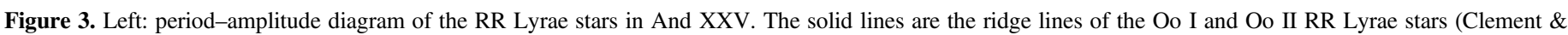

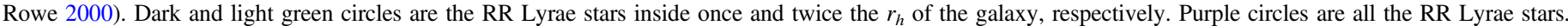

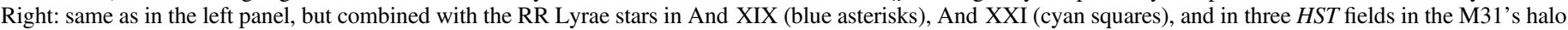
from Sarajedini et al. (2009; gray symbols) and Brown et al. (2004; dark-gray symbols), respectively.

described by Piersimoni et al. (2002). For the sample of RR Lyrae within the $r_{h}$, the reddening is $E(B-V)=0.08 \pm 0.04 \mathrm{mag}$, for the whole sample $E(B-V)=0.05 \pm 0.04$ mag. These values are slightly smaller than those derived by Schlegel et al. (1998), $E(B-V)=0.10 \pm 0.06 \mathrm{mag}$, but are still consistent within $1 \sigma$. To compute the distance to And XXV we adopt the average $V$ magnitude and reddening obtained from the RR Lyrae inside the $r_{h}, M_{\mathrm{V}}=0.54 \pm 0.09 \mathrm{mag}$ for the absolute visual magnitude of RR Lyrae stars with a metallicity of $[\mathrm{Fe} / \mathrm{H}]=$ -1.5 dex (Clementini et al. 2003) and $\frac{\Delta \mathrm{M}_{V}}{\Delta[\mathrm{Fe} / \mathrm{H}]}=$ $-0.214 \pm 0.047 \mathrm{mag} / \mathrm{dex}$ (Clementini et al. 2003; Gratton et al. 2004) for the slope of the RR Lyrae luminosity-metallicity relation. For the metallicity of And XXV we adopt the value $[\mathrm{Fe} / \mathrm{H}]=-1.9 \pm 0.1 \mathrm{dex}$ as derived spectroscopically by Collins et al. (2013). The distance modulus of And XXV derived from the RR Lyrae stars is thus $(m-M)_{0}=24.63 \pm 0.17 \mathrm{mag}$. This estimate is in very good agreement with the result of Richardson et al. (2011), $(m-M)_{0}=24.55 \pm 0.12 \mathrm{mag}$, who used the luminosity of the HB to infer the galaxy distance, but it is more than $1 \sigma$ longer than that obtained by Conn et al. (2012), ( $m$ $M)_{0}=24.33_{-0.21}^{+0.07} \mathrm{mag}$, who used the luminosity of the RGB tip. We suspect that the distance derived in Conn et al. (2012) might be hampered by the uncertain location of the RGB tip, which is scarcely populated in And XXV.

\section{CMD AND STELLAR POPULATIONS}

The CMD of And XXV is shown in Figure 7, where the left panels only contain sources located within the area delimited by the galaxy half-light radius and the ellipticity given by Richardson et al. (2011), the central panel displays sources enclosed between once and twice the $r_{h}$, and the right panel shows the CMD of sources in the whole LBC FOV. We minimized the presence of background galaxies and peculiar sources by only selecting sources with DAOPHOT quality image parameters $-0.35 \leqslant$ sharpness $\leqslant 0.35$ and $\chi<1.5$. Red filled circles are the RR Lyrae stars, green filled triangles are the ACs (the open green triangle is the uncertain $\mathrm{AC} / \mathrm{CC}$ ), the blue pentagon is the unclassified variable, and the ECL is marked by a black asterisk. The main features of the CMD are the RGB and the HB traced by the RR Lyrae stars. The majority of stars filling up the redder part of the CMD at $B-V \sim 1.5-1.7 \mathrm{mag}$ and going beyond $V=22 \mathrm{mag}$, are foreground field objects. V60, the unclassified variable located in this region of the CMD, is most likely a field variable star belonging to the MW field.

The narrow RGB and the poorly populated red HB suggest that And XXV hosts a dominant single old stellar population. In Figure 8 the CMD features and the positions of the variable stars are compared with Padua isochrones obtained using the CMD 2.5 web interface (http://stev.oapd.inaf.it/cgi-bin/cmd) based on models from Bressan et al. (2012). Isochrones for ages from 9 to $13 \mathrm{Gyr}$ and metallicity $Z=0.0003-0.0004$ are good fits to the RGB and the position of the RR Lyrae stars. The red HB is also well-fitted by the isochrones in these age and metallicity ranges.

The only AC discovered within twice the $r_{h}$ of And XXV can either be the result of coalescence in a binary system as old as the RR Lyrae stars, in which mass transfer acted in the last Gyr, or an intermediate young (1-2 Gyr old) star with metallicity in the range $Z=0.0001-0.0006$ (as widely discussed in Papers I and II). The paucity of ACs in And XXV indicates that there is lack of a young/intermediate-age stellar population in this galaxy, when compared to And XIX and And XXI. We selected stars in the CMD of sources in the whole LBC FOV that have color and magnitude in the range of ACs $(0.2 \leqslant B-V \leqslant 0.6,23.0 \leqslant V \leqslant 24.5)$ and we did not find any clear distribution/concentration of stars associated 


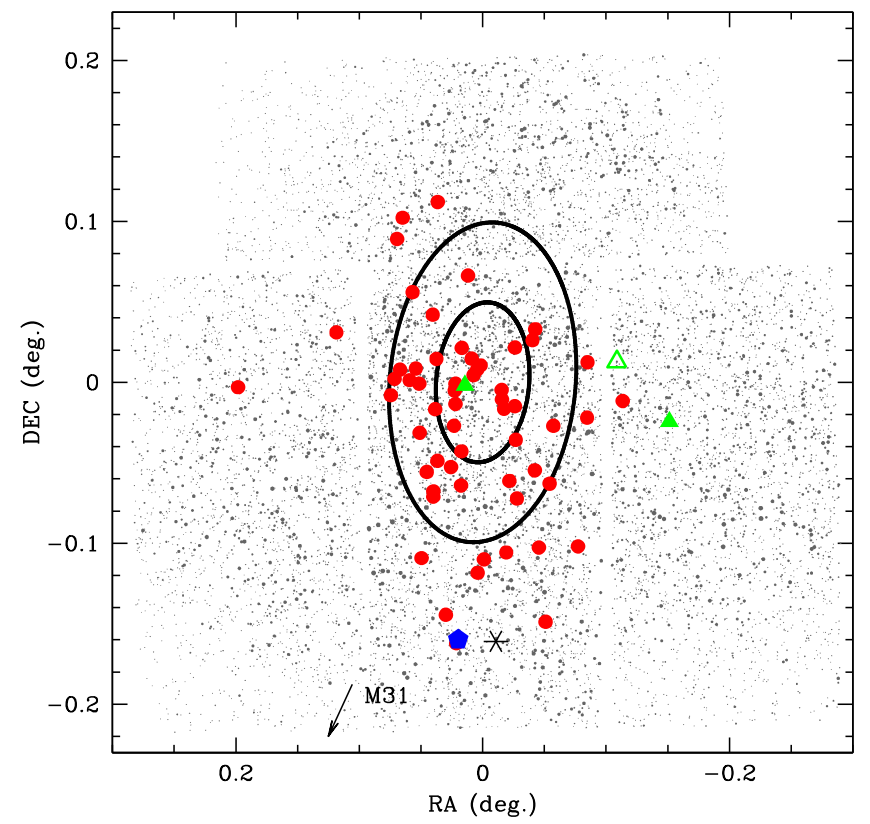

Figure 4. Spatial distribution of the variable stars discovered in the whole LBT FOV. The black ellipses represent the area enclosed by once and twice the $r_{h}$ of And XXV defined by Richardson et al. (2011). Red filled circles mark the RR Lyrae stars, green filled triangles mark the ACs, the open green triangle is the uncertain $\mathrm{AC} / \mathrm{CC}$, the blue pentagon is the unclassified variable, and the black asterisk is the ECL. The arrow points to the direction of M31.

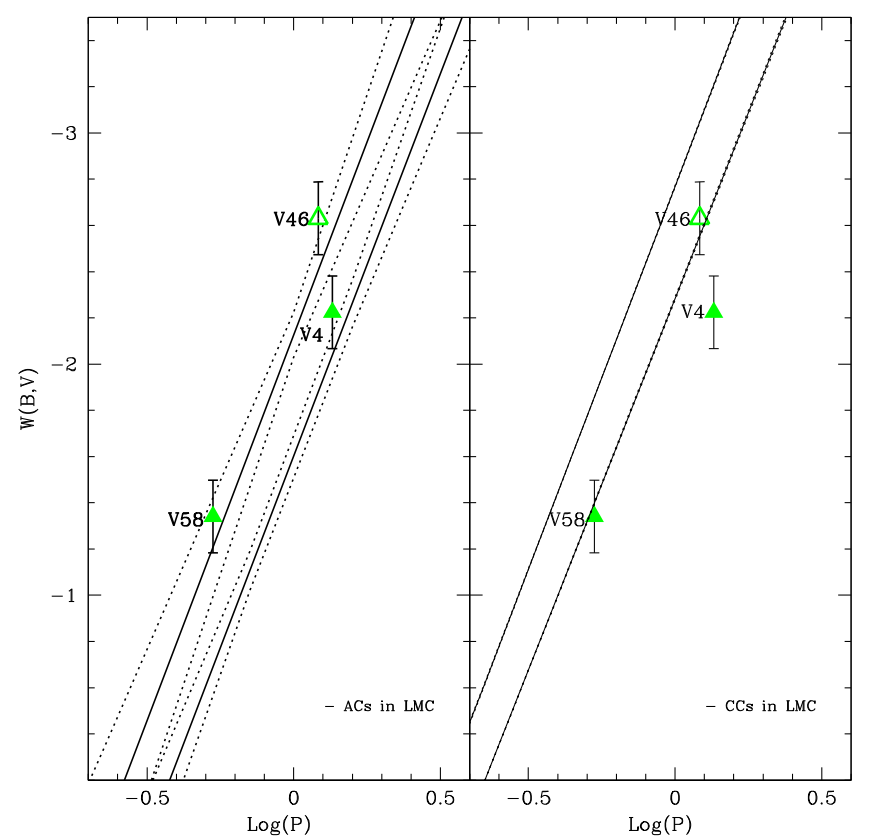

Figure 5. Positions of V4, V46, and V58 with respect to the PW relations for ACs (left panel; Ripepi et al. 2014) and CCs in the LMC (right panel; Jacyszyn-Dobrzeniecka et al. 2016). The $95 \%$ confidence contours are shown as dotted lines. For the CCs, the errors of the fits are very small and the confidence contours are very close to the fits.

with this selection. The star formation in And XXV most likely was quenched by some external event such as, for instance, tidal stripping by large nearby galaxies like the NGC 147/ NG 185 pair (see the next section) or from M31 itself. In this scenario, the presence of at least one $\mathrm{CC}$ and one $\mathrm{AC}$

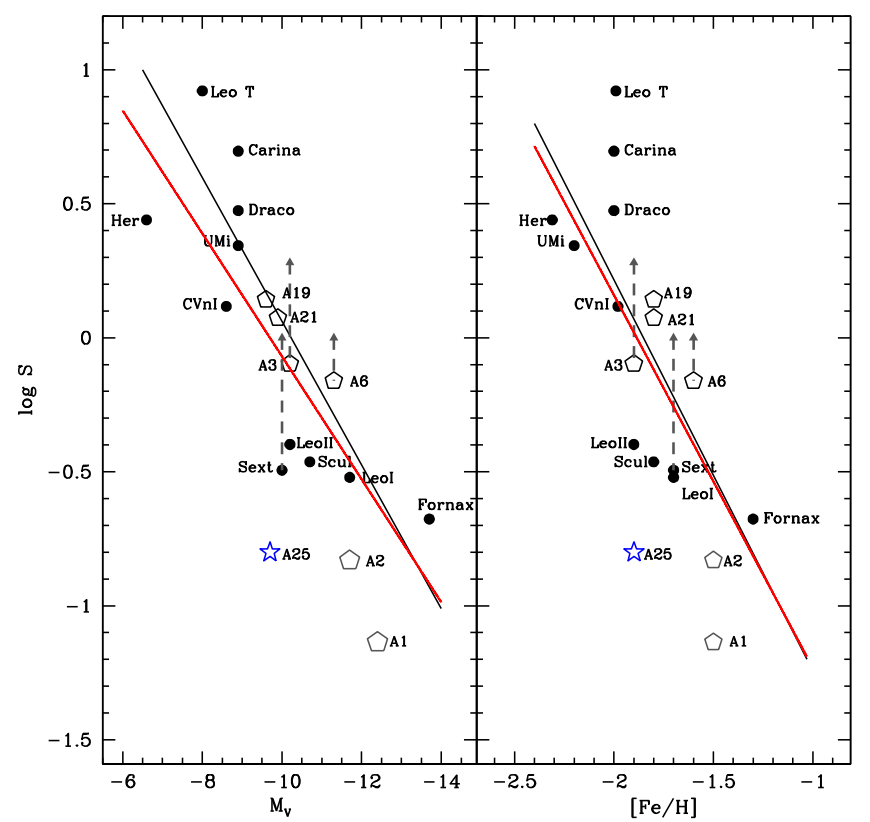

Figure 6. Left: specific frequency of ACs in dwarf satellites of the MW (filled circles) and M31 (open pentagons) vs. absolute visual magnitude. And XXV is represented by the blue star. Black lines are the least-squares fits of ACs by Pritzl et al. (2005) while the red solid lines mark the least-squares fits we have been obtaining using only ACs confirmed by the comparison with the PW relations for ACs (see the text for details). Right: same as in the left panel, but vs. metallicity of the parent galaxy.

off-centered with respect to And XXV could indicate that a mild recent activity has been triggered from the outside.

\subsection{Projected Spatial Distributions}

RGB stars were selected from the CMD in the right panel of Figure 7, showing stars contained in the whole LBT FOV. The selection performed is marked with a magenta region in Figure 7. The RGB isodensity contours are shown in the left panel of Figure 9 and reveal very interesting features of And XXV structure.

Moving outward from the galaxy center, the outermost contours appear to be elongated toward the positions of NGC 147 and NGC 185, and the major axis direction of the isodensity contours changes, and points toward the direction of these two galaxies. Since isodensity twisting is mainly due to tidal effects (di Tullio 1979; Johnston et al. 2002), we suggest that And XXV is likely experiencing tidal stirring from the relatively nearby couple NGC $147 / \mathrm{NGC} 185$ on one side and from M31 in the orthogonal direction. Perhaps And XXV is infalling the M31 halo for the first time and feeling both the tidal force of the NGC 147/NGC 185 couple and that of the massive host galaxy M31. Projected distances to NGC 147/ NGC 185 and M31 are $295 \mathrm{kpc}$ and $\sim 143 \mathrm{kpc}$, respectively, (adopting distances reported in Conn et al. 2012 for these systems). The right panel of Figure 9 shows the spatial distribution of the HB stars. This part of the CMD is heavily contaminated by background unresolved galaxies. Nevertheless it is still possible to see some of the features traced by the RGB isodensities. A protrusion of HB stars extending in the direction of the M31 center highlights the tidal interaction between And XXV and M31. 


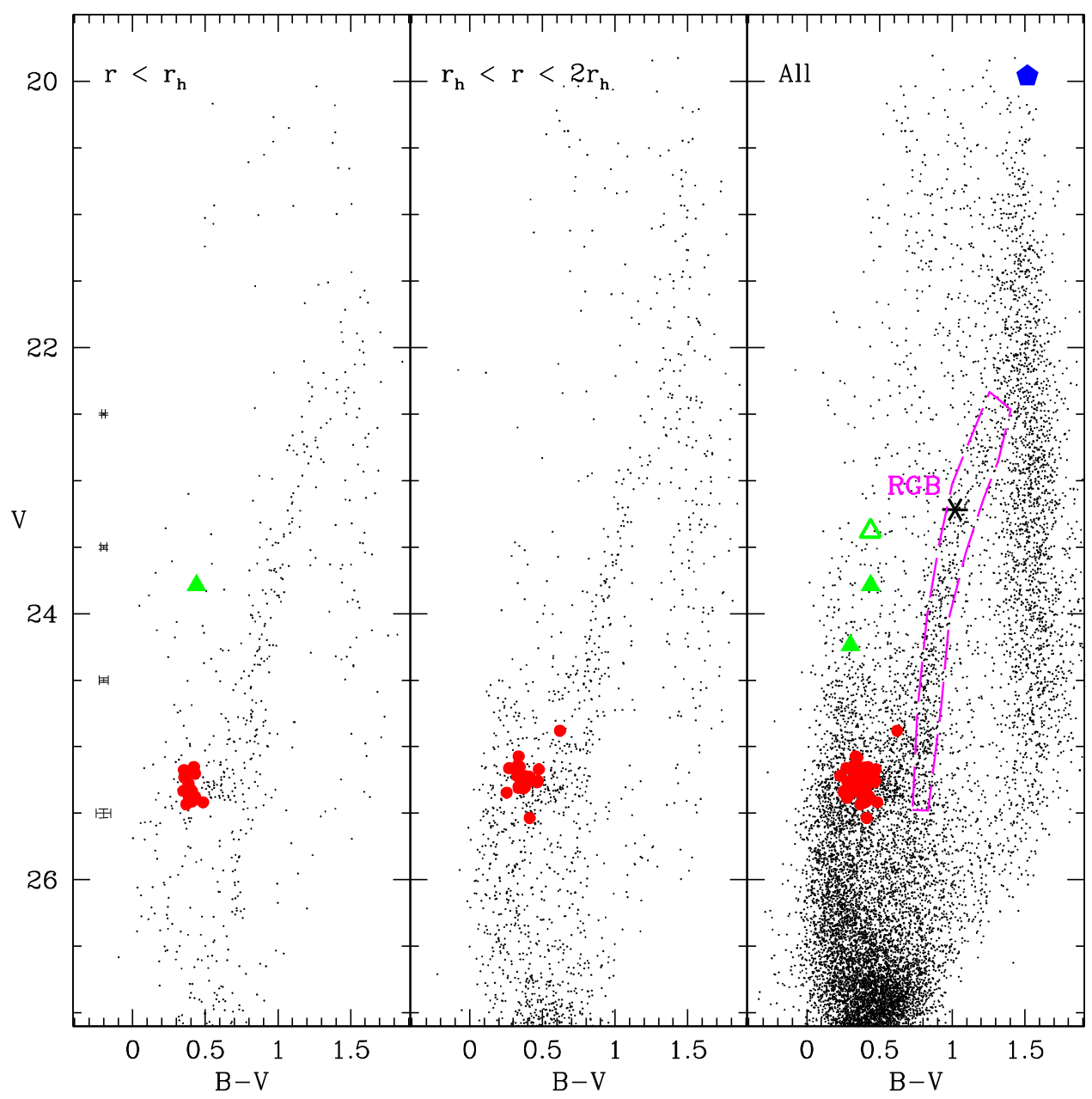

Figure 7. Left: CMD of sources in our photometric catalog with $-0.35 \leqslant$ sharpness $\leqslant 0.35$ and with $\chi<1.5$ and located within the area delimited by the galaxy half-light radius and the ellipticity by Richardson et al. (2011). Red circles mark the RR Lyrae stars, while green triangles are the ACs. Center: same as in the left panel, but within the area between once and twice the galaxy $r_{h}$. Right: same as in the left panel, but considering sources in the whole LBC FOV. The black asterisk is the ECL, the blue pentagon is the unclassified variable, and the open green triangle is the uncertain $\mathrm{AC} / \mathrm{CC}$.

\section{GEP I: AND XXV'S CENTER OR A NEW GLOBULAR CLUSTER ORBITING AND XXV OR M31?}

Visual inspection of the deep image obtained by stacking all the $B$-band LBC frames (for a total of $9.4 \mathrm{hr}$ integration time) has revealed a spherical assembly of stars near And XXV's photometric center, which we have named Gep I. ${ }^{8}$ This serendipitously discovered concentration of stars is centered around coordinates R.A. $=00: 30: 10.579$ decl. $=+46: 51: 05.58$ and is of $\sim 12$ arcsec in diameter. A snapshot of Gep I from our deep LBT $B$-band image is shown in Figure 10. In the discovery paper, Richardson et al. (2011) note that And XXV falls close to the 2 arcmin gaps among CCDs in their observations (see Figure 2 of Richardson et al. 2011) significantly hampering the determination of And XXVs centroid, for which they estimate a quite large $\sim 12$ arcsec uncertainty. The difference between Gep I and And XXV center coordinates is $\sim 11$ arcsec, hence, Gep I is within the error box of Richardson et al.'s (2011) center coordinates of the galaxy.

The stellar concentration could either be And XXV's actual center or a new star cluster belonging to And XXV or M31. Indeed, there is no known Globular Cluster (GC) in the

\footnotetext{
8 In the memory of a young colleague of ours, Geppina Coppola, who prematurely passed away.
}

Revised Bologna Catalog of the M31 GCs (Galleti et al. 2004), nor any other known extended source within $\sim 20$ arcsec of Gep I center coordinates. Integrated photometry of Gep I was performed using the Graphical Astronomy and Image Analysis Tool, starting from the center of the cluster and with circular apertures ranging from 1 to 20 arcsec radius. With this method we estimated the half-light radius of Gep I to be $r_{h} \sim 6 \operatorname{arcsec}$ and its integrated magnitudes, computed within twice the $r_{h}$, are $B \sim 20.6 \mathrm{mag}$ and $V \sim 20.0 \mathrm{mag}$, respectively. Assuming the distance modulus and reddening of And XXV, we obtain absolute magnitudes of $M_{B} \sim-4.2 \mathrm{mag}$ and $M_{V} \sim-4.9 \mathrm{mag}$, respectively. The $r_{h}$ of Gep I at the distance of And XXV corresponds to $\sim 25 \mathrm{pc}$ in linear extension. Radius and absolute $V$ magnitude place Gep I in the region of the $M_{V}-r_{h}$ plane that seems forbidden to ordinary GCs. Only the M31 Extended Clusters (ECs, Huxor et al. 2011) and the least luminous among the MW Palomar GCs are found to lie in this region (see Figure 11 of Huxor et al. 2011), along with the nuclei of dwarf elliptical galaxies. This leaves open the possibility that Gep I could indeed be the center of And XXV.

There are few stars that our photometry could resolve in this cluster and their position in the CMD is shown in the left panel of Figure 11. This CMD was performed by taking all the objects in a circular area of 12 arcsec in radius from the center 


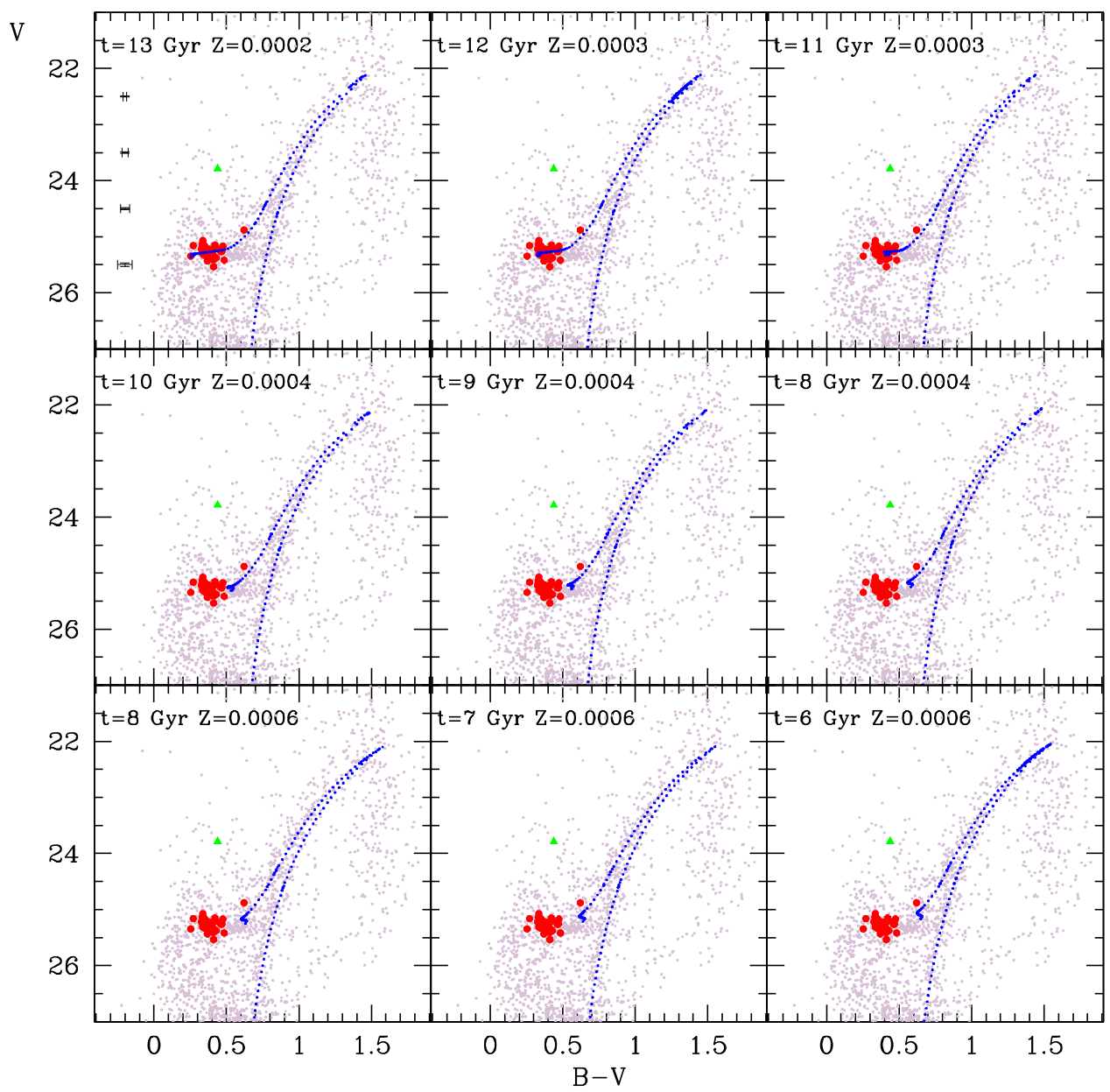

Figure 8. CMD of sources contained within twice the $r_{h}$ of And XXV, overlaid by Padua stellar isochrones (Bressan et al. 2012) with different ages (from $t=13$ to $6 \mathrm{Gyr}$ ) and metallicities $(Z=0.0003, Z=0.0004$ and $Z=0.0006$; from top to bottom). Red filled circles are RR Lyrae stars, and the green filled triangle is the $\mathrm{AC}(\mathrm{V} 4)$.
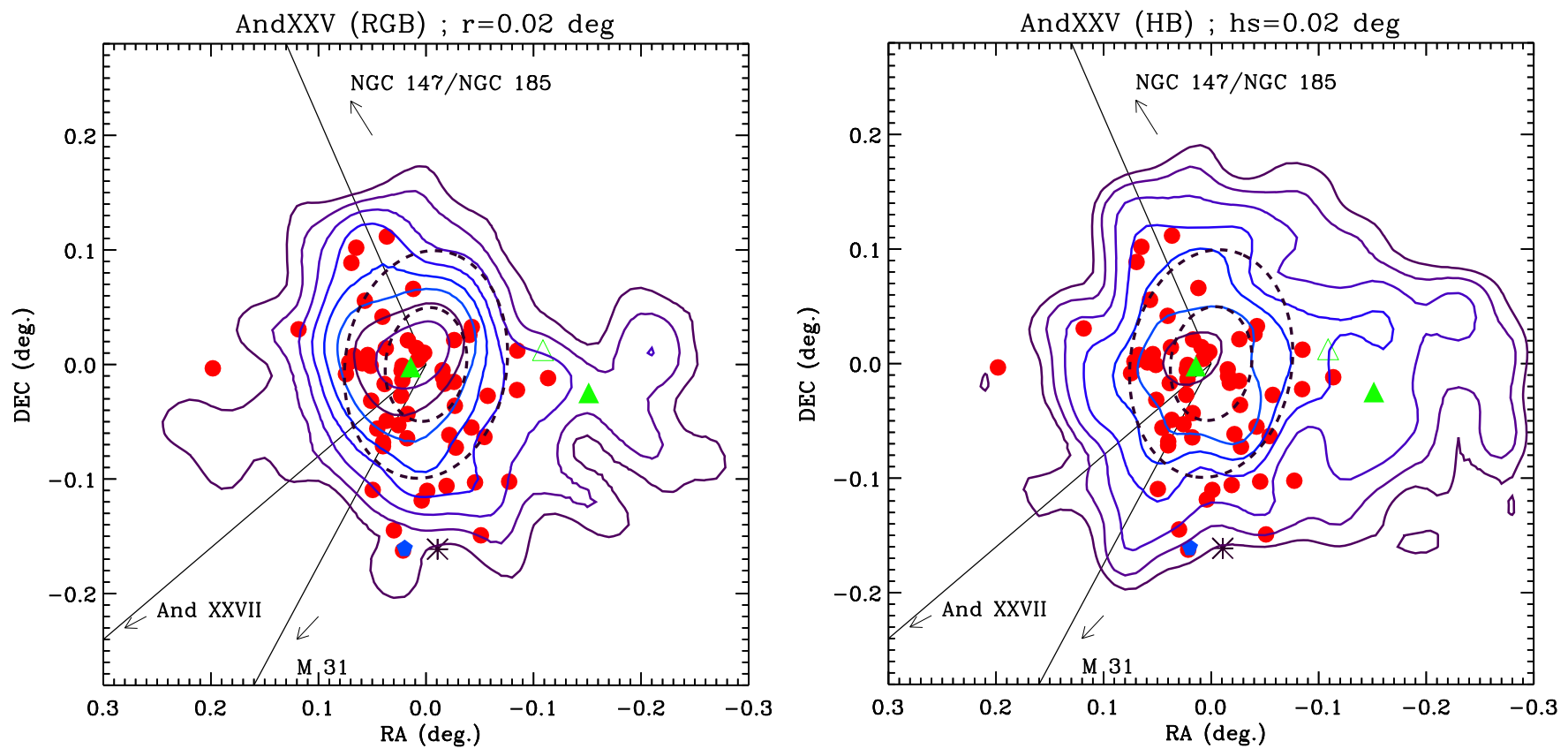

Figure 9. Left: isodensity contours of RGB stars in And XXV. R.A. and decl. are the differential coordinates computed from the center of the galaxy given by Richardson et al. (2011). The black dashed ellipses mark the areas within once and twice the $r_{h}$. The variable stars are shown with different colors and symbols [red circles are for RR Lyrae stars, and green filled triangles are for ACs (the open green triangle is for the uncertain AC/CC), black asterisks are for the ECL, the blue pentagon is for the unclassified variable]. Right: same as in the left panel but for And XXV HB stars. 

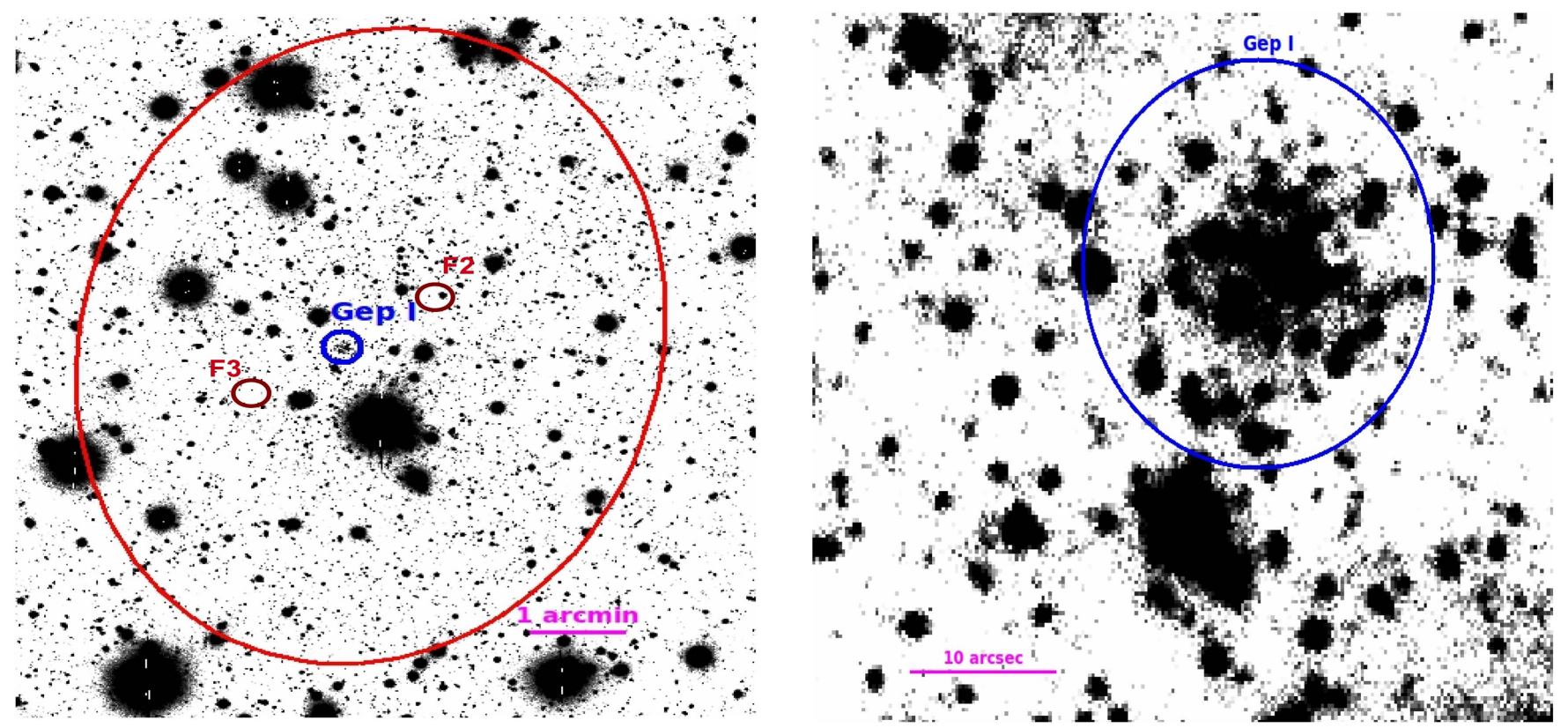

Figure 10. Left: part of the deep image obtained by stacking all $B$-band frames. The position of Gep I is highlighted with a blue circle. The positions of the comparison fields F2 and F3 are marked with red circles. The concentration of stars is near the center of And XXV; the red ellipse shows the area delimited by once the $r_{h}$. North is up and east to the left. Right: a closed in view of Gep I.

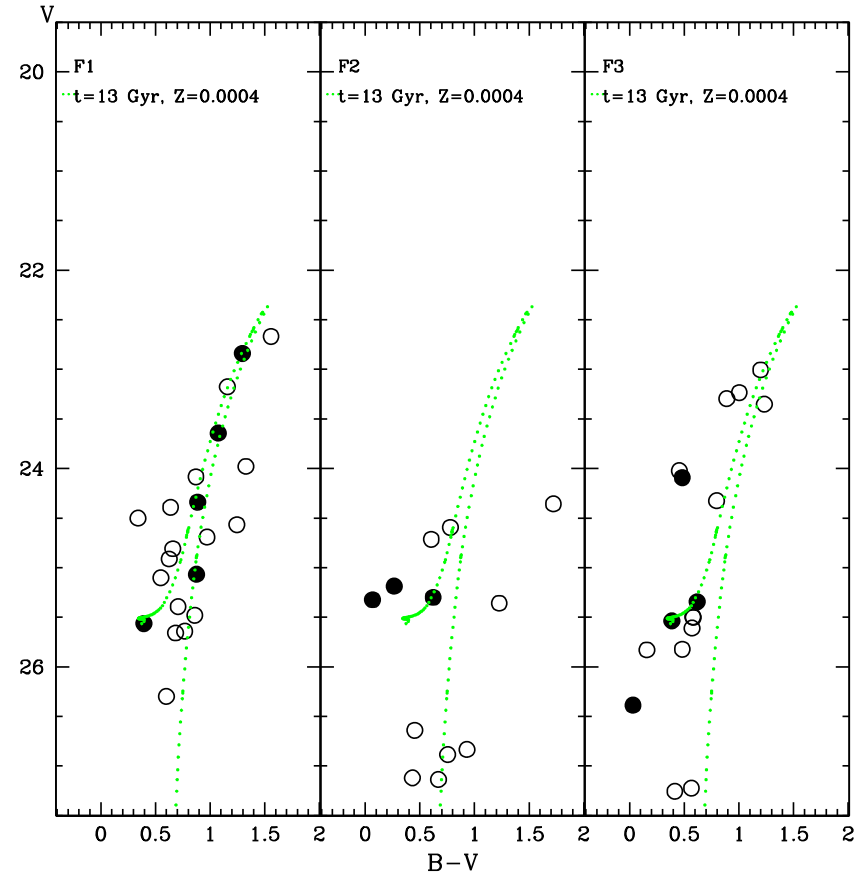

Figure 11. Left: CMD of sources in a circular area within a radius of 12 arcsec from the center of Gep I (field F1). Filled circles are sources in our LBC photometric catalog selected using the DAOPHOT $\chi$ and sharpness parameters $(-0.35 \leqslant$ sharpness $\leqslant 0.35$ and $\chi<1.5)$. The green dashed line is an isochrone with $t=13 \mathrm{Gyr}$ and $Z=0.0004$ from Bressan et al. (2012). Center: same as in the left panel but for sources in F2. Right: same as in the left panel but for sources in F3.

of the stellar association (field F1). These stars are mostly red giants, with only one or two HB stars. Their location in the CMD is compatible with an old population placed at a Heliocentric distance of $\sim 750-800 \mathrm{kpc}$. The center and right panels of Figure 11 show, for a comparison, the CMDs of sources in two circular regions F2 (central panel) and F3 (right panel) of a radius equal to twice the estimated $r_{h}$ of Gep I, but centered $\sim 1$ arcmin to the northwest and $\sim 1$ arcmin to southeast with respect to the F1 center, respectively. The positions of F2 and F3 in the LBC FOV are marked with red circles in Figure 10. The CMDs of fields F2 and F3 show no clear evidence of a single old stellar population similar to the one observed in field F1, thus confirming that in F1 there is a real concentration of old stars. Clearly, this ground-based CMD of Gep I is severely incomplete.

To unveil the nature of Gep I we have proposed follow up Hubble Space Telescope observations in order to resolve and characterize its stellar populations and precisely measure its distance. If the concentration of stars is proven to be a GC belonging to And XXV, the galaxy would be one of the few dSphs in the Local Group known to host GCs, after Fornax and Sagittarius.

Moreover, the position of this putative GC very close to the center of And XXV (according to Richardson et al. 2011 coordinates) would resemble the case of M54, which is in the core of its parent galaxy, the Sagittarius dSph, according to Monaco et al. (2005). Very recently a stellar cluster was detected near the center of the Eridanus II dwarf galaxy by Crnojevic et al. (2016). The authors claim that Eridanus II is the faintest galaxy to host a stellar cluster. In the same vein, And XXV could be the faintest galaxy of the M31 complex to host a stellar cluster.

Zaritsky et al. (2016) have suggested that most GCs may be hosted by undetected faint galaxies. Our discovery in And XXV, along with Crnojević et al.'s (2016) discovery of the cluster in Eridanus II, may lend support to Zaritsky et al.'s (2016) claim and provide hints on the connection between GCs and dwarf galaxies.

\section{SUMMARY AND CONCLUSIONS}

Based on $B, V$ time series photometry obtained with LBCs at LBT, we have discovered 62 variable stars in the field of 
Table 3

Properties of the Variable Stars in the Andromeda Satellite Galaxies

\begin{tabular}{|c|c|c|c|c|c|c|c|}
\hline Name & $N(\mathrm{RRab}+\mathrm{RRc})$ & $\left\langle P_{\mathrm{ab}}\right\rangle$ & $f_{c}$ & $N(\mathrm{AC})$ & $N$ (AC) Confirmed $^{\mathrm{a}}$ & Member & Reference \\
\hline And I & $72+26$ & 0.57 & 0.26 & $1 ?$ & 0 & yes & (1) \\
\hline And II & $64+8$ & 0.57 & 0.11 & 1 & 0 & no & (2) \\
\hline And III & $39+12$ & 0.66 & 0.23 & $5 ?$ & 2 & yes & (1) \\
\hline And VI & $91+20$ & 0.59 & 0.18 & 6 & 4 & no & (3) \\
\hline And XI & $10+5$ & 0.62 & 0.33 & 0 & 0 & yes & (4) \\
\hline And XIII & $12+5$ & 0.66 & 0.30 & 0 & 0 & yes & (5) \\
\hline And XVI & $3+6$ & 0.64 & 0.33 & 0 & 0 & no ${ }^{b}$ & $(5,6)$ \\
\hline And XIX & $23+8$ & 0.62 & 0.26 & 8 & 8 & no & (7) \\
\hline And XXI & $37+4$ & 0.63 & 0.10 & 9 & 9 & no & (8) \\
\hline And XXV & $46+11$ & 0.60 & 0.19 & 2 & 1 & yes & (9) \\
\hline
\end{tabular}

Notes.

a See Section 3.2 .

${ }^{\mathrm{b}}$ Offset by $8 \mathrm{kpc}$ from the GPoA.

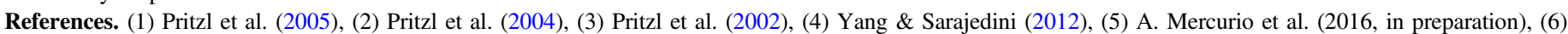
Monelli et al. (2016), (7) Cusano et al. (2013), (8) Cusano et al. (2015), (9) this work.

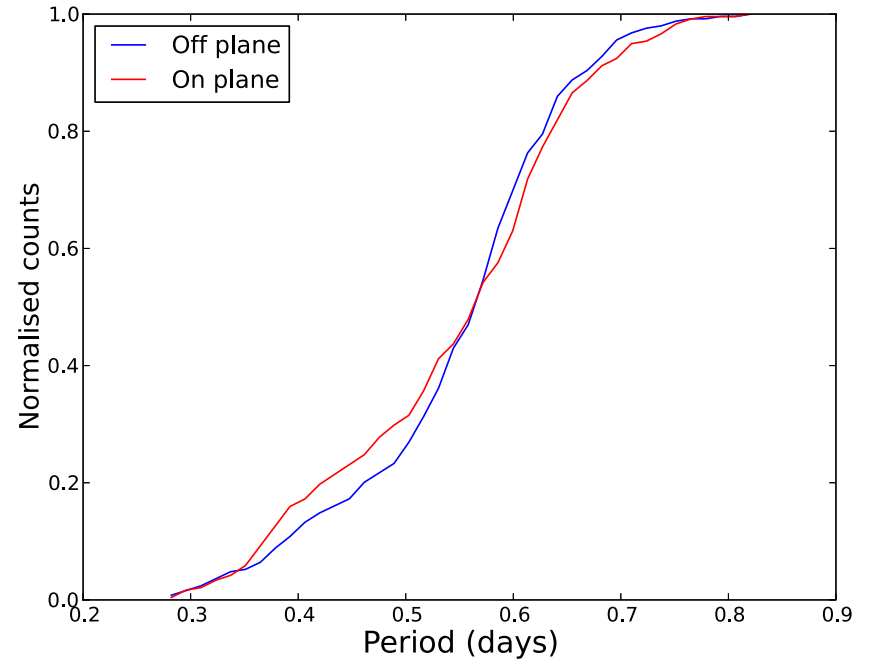

Figure 12. Cumulative period distribution of the RR Lyrae stars in M31 satellite galaxies on and off the GPoA.

And XXV dSph, of which 57 are RR Lyrae stars, 3 are ACs, 1 is an ECL, and 1 is an unclassified variable. The average period of the RRab stars classifies this galaxy as an Oosterhoffintermediate object. The comparison of the galaxy CMD with isochrones and the population of variable stars is compatible with a single old (9-13 Gyr) and metal-poor ([Fe/H] $\sim-1.8$ dex) stellar generation being the predominant component of And XXV. In the field of And XXV we have detected a spherical concentration of stars near the galaxy center that could either be a candidate globular cluster or the center of the galaxy. And XXV is the first of the M31 satellites that we have investigated so far to lay on the Great plane of Andromeda (GPoA) defined by Ibata et al. (2013). The stellar populations detected in And XXV differ somehow from the stellar populations that we have identified in And XIX and And XXI, which both lay off the GPoA. In particular, in And XXV there is lack of an intermediate/young stellar population, compared to And XIX and And XXI, and a very small number of ACs. Besides And XXV, only four other M31 satellites on the GpoA have been investigated so far to detect variable stars and have a metallicty $[\mathrm{Fe} / \mathrm{H}] \leqslant-1.5 \mathrm{dex}$ (which is the condition to generate ACs, see, e.g., Marconi et al. 2004), namely: And I, And III (Pritzl et al. 2005), And XI, and And XIII (Yang \& Sarajedini 2012). Pritzl et al. (2005) found no ACs in And I and two ACs in And III (following our re-analysis in Section 3.2), while in And XI and And XIII no ACs were detected. Although at present the statistic is still rather meager (5 out of 15 satellites laying on the plane) it seems that galaxies on the GPoA have no or very few ACs, hence they host no or very few intermediate-age stars. ${ }^{9}$

We have compared the pulsation properties of the RR Lyrae population in the M31 dSph satellites on and off the GPoA. The M31 dSphs that have been studied for variability are listed in Table 3 along with their membership to the GPoA and the characteristics of their RR Lyrae populations. The average periods of RRab stars for galaxies on and off the plane are $\left\langle P_{\mathrm{ab}}\right\rangle=0.62 \pm 0.07$ day and $\left\langle P_{\mathrm{ab}}\right\rangle=0.60 \pm 0.06$ day, respectively. The fraction of $\mathrm{RRc}$ to the total number of $\mathrm{RR}$ Lyrae stars is $f_{c}=0.31$ and $f_{c}=0.23$ for on-plane and off-plane satellites. Both populations are thus compatible with an Oo-Int classification, but with a slight tendency toward Oo-II type for the on-plane satellites. In Figure 12 we plot the cumulative period distribution of the RR Lyrae stars in the two samples. A twosample Kolmogorov-Smirnov (K-S) test was performed to check if there are significant differences between the two populations. The $p$-value of the $\mathrm{K}-\mathrm{S}$ test is $p=0.36$ and at this level of the investigation we cannot reject the hypothesis that the two RR Lyrae populations have very similar pulsation properties. For the ACs' distribution, there is a clear difference, considering that the total number of ACs for on-plane galaxies is 3, while this number for the off-plane galaxies is 21 (see Table 3 column 6). From the variable stars we can thus perhaps draw the first picture of the M31 satellite complex, in which all satellites had a common ancient star formation episode about 10-12 Gyr ago that led to the formation of RR Lyrae stars. After that, the evolution history of on-plane and off-plane satellites started to differentiate and only the latter were able to retain enough gas to produce an intermediate-age stellar population that also gave rise to significant numbers of ACs.

\footnotetext{
9 We note that And XVI, a galaxy located at a projected distance of $d_{M 31}=323 \mathrm{kpc}$ from the M31 center, but offset by only $8 \mathrm{kpc}$ from the GPoA (Pawlowski et al. 2013), also does not seem to contain ACs (Monelli et al. 2016).
} 
We warmly thank P. Montegriffo for the development and maintenance of the GRATIS software. Financial support for this research was provided by PRIN INAF 2010 (PI: G. Clementini) and by Premiale LBT 2013. The LBT is an international collaboration among institutions in the United States, Italy, and Germany. LBT Corporation partners are The University of Arizona on behalf of the Arizona university system; Istituto Nazionale di Astrofisica, Italy; LBT Beteiligungsgesellschaft, Germany, representing the Max-Planck Society, the Astrophysical Institute Potsdam, and Heidelberg University; The Ohio State University; and The Research Corporation, on behalf of The University of Notre Dame, University of Minnesota, and University of Virginia. We acknowledge the support from the LBT-Italian Coordination Facility for the execution of observations, data distribution, and reduction.

Facility: LBT.

\section{REFERENCES}

Bailey, S. I. 1902, AnHar, 38, 1

Bressan, A., Marigo, P., Girardi, L., et al. 2012, MNRAS, 427, 127

Brown, T. M., Ferguson, H. C., Smith, E., et al. 2004, AJ, 127, 2738

Bullock, J. S., \& Johnston, K. V. 2005, ApJ, 635, 931

Cardelli, J. A., Clayton, G. C., \& Mathis, J. S. 1989, ApJ, 345, 245

Catelan, M. 2009, Ap\&SS, 320, 261

Clement, C. M., \& Rowe, J. 2000, AJ, 120, 2579

Clementini, G., Di Tomaso, S., Di Fabrizio, L., et al. 2000, AJ, 120, 2054

Clementini, G., Gratton, R., Bragaglia, A., et al. 2003, AJ, 125, 1309

Collins, M. L. M., Chapman, S. C., Rich, R. M., et al. 2013, ApJ, 768, 172

Collins, M. L. M., Chapman, S. C., Rich, R. M., et al. 2014, ApJ, 783, 7

Conn, A. R., Ibata, R. A., Lewis, G. F., et al. 2012, ApJ, 758, 11

Crnojević, D., Sand, D. J., Zaritsky, D., et al. 2016, ApJ, 824L, 14
Cusano, F., Clementini, G., Garofalo, A., et al. 2013, ApJ, 779, 7

Cusano, F., Garofalo, A., Clementini, G., et al. 2015, ApJ, 806, 200

di Tullio, G. A. 1979, A\&AS, 37, 591

Galleti, S., Federici, L., Bellazzini, M., Fusi Pecci, F., \& Macrina, S. 2004 A\&A, 416, 917

Gratton, R. G., Bragaglia, A., Clementini, G., et al. 2004, A\&A, 421, 937

Huxor, A. P., Ferguson, A. M. N., Tanvir, N. R., et al. 2011, MNRAS, 414,770

Ibata, R. A., Lewis, G. F., Conn, A. R., et al. 2013, Natur, 493, 62

Jacyszyn-Dobrzeniecka, A. M., Skowron, D. M., Mróz, P., et al. 2016, AcA, 66,149

Johnston, K. V., Choi, P. I., \& Guhathakurta, P. 2002, AJ, 124, 127

Marconi, M., Fiorentino, G., \& Caputo, F. 2004, A\&A, 417, 1101

Martin, N. F., Ibata, R. A., McConnachie, A. W., et al. 2013, ApJ, 776, 80

McGaugh, S., \& Milgrom, M. 2013, ApJ, 775, 139

Monaco, L., Bellazzini, M., Ferraro, F. R., \& Pancino, E. 2005, MNRAS, 356, 1396

Monelli, M., Martínez-Vázquez, C. E., Bernard, E. J., et al. 2016, ApJ, 819,147

Oosterhoff, P. T. 1939, Obs, 62, 104

Pawlowski, M. S., Kroupa, P., Jerjen, H., et al. 2013, MNRAS, 435, 1928

Piersimoni, A. M., Bono, G., \& Ripepi, V. 2002, AJ, 124, 1528

Pritzl, B. J., Armandroff, T. E., Jacoby, G. H., \& Da Costa, G. S. 2002, AJ, 124,1464

Pritzl, B. J., Armandroff, T. E., Jacoby, G. H., \& Da Costa, G. S. 2004, AJ, 127,318

Pritzl, B. J., Armandroff, T. E., Jacoby, G. H., \& Da Costa, G. S. 2005, AJ, 129,2232

Richardson, J. C., Irwin, M. J., McConnachie, A. W., et al. 2011, ApJ, 732, 76

Ripepi, V., Marconi, M., Moretti, M. I., et al. 2014, MNRAS, 437, 2307

Sarajedini, A., Mancone, C. L., Lauer, T. R., et al. 2009, AJ, 138, 184

Schlegel, D. J., Finkbeiner, D. P., \& Davis, M. 1998, ApJ, 500, 525

Stetson, P. B. 1987, PASP, 99, 191

Stetson, P. B. 1994, PASP, 106, 250

Yang, S.-C., \& Sarajedini, A. 2012, MNRAS, 419, 1362

Zaritsky, D., Crnojević, D., \& Sand, D. J. 2016, ApJL, 826, L9 